\title{
The space distribution and transfer of positioning errors from actuators to the TCP point of parallel mechanism
}

\author{
Vladimír Bulej ${ }^{1, *}$, Ján Stanček ${ }^{1}$, Ivan Kuric ${ }^{1}$, Ivan Zajacko ${ }^{1}$ \\ ${ }^{1}$ University of Zilina, Faculty of Mechanical Engineering, Department of Automation and Production \\ Systems, Univerzitna 1, 01026 Zilina, Slovakia
}

\begin{abstract}
The article deals with the analysis of the transfer and distribution of inaccuracies from actuators to the position of the point TCP on one of these machines. The real functional model of the mechanism was developed at the authors' workplace. The analyses of the workspace were carried out on CAD model. The results should be used for simulation of mechanism's behaviour, structural optimization as well as for generating of control algorithms for real functional prototype.
\end{abstract}

Keywords: parallel kinematic structure, hybrid kinematic structure, simulation and modelling, inaccuracies distribution in workspace

\section{Introduction}

High speed cutting (HSC) has its own special place in field of machining. High-speed manipulation is a visible similar trend in robotics. It appears that for both areas the machines with parallel and hybrid kinematic structure fit better than machines with conventional serial kinematics. These mechanisms are characterized above all by higher stiffness and higher dynamic parameters (mainly due to reduced moving mass and inertia) [1-4]. One of the well-known fully parallel mechanisms is called Hexapod or GoughStewart platform. Hexapod is multi-axis mechanism capable of full six degrees of freedom (DOFs) motion. Few years ago a research group at the University of Zilina started to deal with area of parallel mechanisms as well. During this period there were designed some mechanism concepts and different kind of simulation software for these mechanisms [5].

Main aim of this article is the analysis of space distribution and transfer of positioning errors from actuators to the TCP point of mechanism with parallel kinematic structure, concretely machine tool prototype called Hexapod $Z U$. When the end effector of hexapod is positioning in any axis, resulting motion is composed of several simple extensions of each separate actuator. It seems to be important to study more detailed the properties of this type of mechanisms to understand their behaviour. This article contains the first analysis results, which was carried out on 3D CAD model of the mechanism in software Pro Engineer Wildfire 4.

\footnotetext{
* Corresponding author: vladimir.bulej@fstroj.uniza.sk

Reviewers: Andrej Czán, Ksawery Szykiedans
} 


\section{Evaluation of the positioning error space distribution}

The evaluation of the positioning error of a manipulator is a fundamental step in the design process if accuracy and stiffness are the guidelines for the mechanical project, as is the case for machining or assembly manipulators. In most of these works of these works, the analytic model is exploited to evaluate the stiffness of the manipulator in a series of configurations inside the workspace [6].

We created a three-dimensional grid of points (figure 1) for the distribution analysis of positioning errors by positioning in three axes [7]. This grid represents selected set of positions of moving platform where we take measurements as well as where we apply the inaccuracies into the arms. The inaccuracy was defined as an extension of $1 \mathrm{~mm}$ implemented on one or more arm / arms. The result is an output value of final deviation between the required position of TCP point and the position of TCP affected by an error. The final deviation can be divided into each axis separately.

The grid size was chosen according to maximize workpiece size. The workpiece centre point is aligned with the centre of working table. Within the machine tool workspace was chosen a main coordinate system whose location is given by the axis of the machine tool and the top horizontal surface of a workpiece. Then we defined two horizontal planes, which are labelled as level 1 and level 2 and in each were defined 9 points. Both of them, level 1 as well as level 2 are parallel with the plane XY.

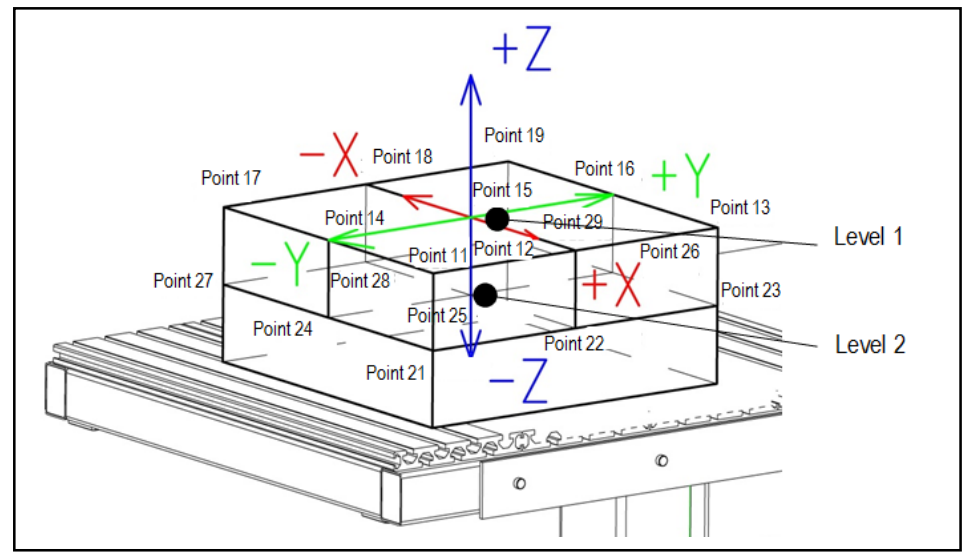

Fig. 1. The workspace divided into the 3D grid of points placed in 2 levels [7]

\subsection{Reference acting values in all points}

At the beginning it was necessary to determine the initialization values of extension for each linear actuator for all defined points (figure 1 and figure 2). These values are considered as reference positions. To this reference values will be continuously added the specified increment which will represent the source of positioning errors (input positioning error). Consequently will be measured the deviation from the expected position of TCP point (output positioning error) in axis $\mathrm{X}, \mathrm{Y}$ and $\mathrm{Z}$ as well as the total space error.

The value of input positioning error was determined as $+2 \mu \mathrm{m}$ unit displacement (positive extension of actuator) with respect to the predicted accuracy of final mechanism. We decided for application of electromechanical linear actuators Exlar IX30-1602 with a preloaded planetary roller screw system as arms in design of tested mechanism. These actuators offer the high load carrying capacity (nominal more than $4000 \mathrm{~N}$ per actuator) as well as up to 15 times the life of an equivalent ball screw. 


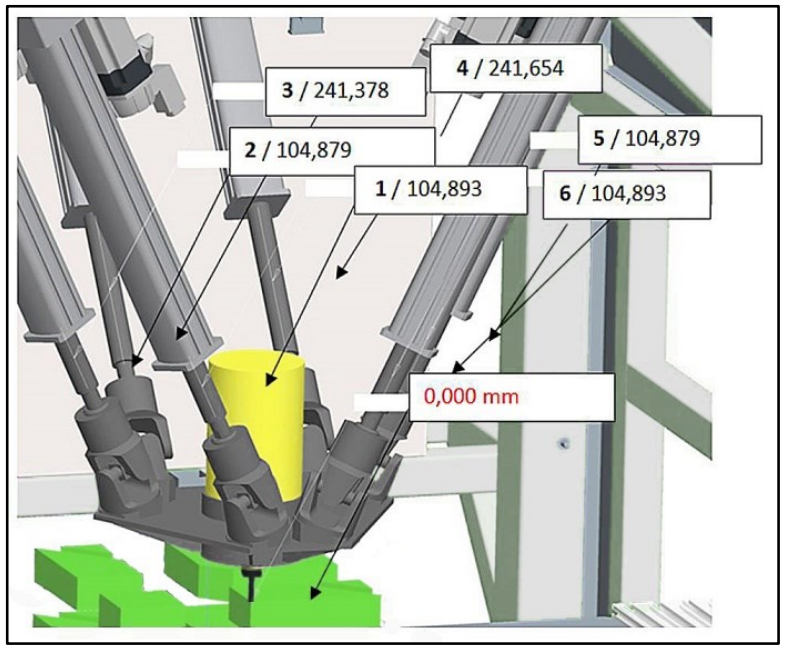

Fig. 2. Example how to reach the acting values (linear extensions) with respect to the position of moving platform (here is shown the case when moving platform is located in point 12)

The defined errors will be gradually applied on actuators according to following schema: error is applied on arm number 1; error is applied on arms number 1 and 2; then 1, 2 and 3 up to case when error is applied on all of the arms. There were done 2 other simulations (will be not mentioned in article) with different values of applied input positioning error: $+1 \mathrm{~mm}$ and $+1 \mu \mathrm{m}$. First of them were used only to illustrate the principle how are positional errors distributed in the $3 \mathrm{D}$ space and their transfer from actuators to the TCP point.

Table 1. Extension of each arms (from 1 to 6 ) to achieve separate grid points

\begin{tabular}{|c|c||c|c|c|c|c|c|}
\hline \multirow{2}{*}{ Level } & \multirow{2}{*}{ Point } & \multicolumn{7}{|c|}{ Extension of arm number: (mm) } \\
\cline { 3 - 8 } & & $\mathbf{1}$ & $\mathbf{2}$ & $\mathbf{3}$ & $\mathbf{4}$ & $\mathbf{5}$ & $\mathbf{6}$ \\
\hline \hline 1 & 11 & 39,595 & 39,588 & 260,196 & 260,544 & 206,071 & 206,092 \\
\hline 1 & 12 & 104,893 & 104,879 & 241,378 & 241,654 & 104,879 & 104,893 \\
\hline 1 & 13 & 206,920 & 206,710 & 260,480 & 260,558 & 39,588 & 39,595 \\
\hline 1 & 14 & 68,271 & 68,279 & 152,490 & 152,635 & 230,169 & 230,176 \\
\hline 1 & 15 & 131,577 & 131,577 & 131,577 & 131,577 & 131,577 & 131,577 \\
\hline 1 & 16 & 230,176 & 230,169 & 152,492 & 152,651 & 68,279 & 68,271 \\
\hline 1 & 17 & 139,283 & 139,305 & 77,419 & 77,380 & 290,772 & 290,767 \\
\hline 1 & 18 & 198,128 & 198,141 & 54,545 & 54,486 & 198,141 & 198,128 \\
\hline 1 & 19 & 290,767 & 290,772 & 77,392 & 77,398 & 139,305 & 139,283 \\
\hline 2 & 21 & 137,345 & 137,339 & 339,403 & 339,615 & 289,078 & 289,097 \\
\hline 2 & 22 & 196,304 & 196,290 & 321,912 & 322,049 & 196,890 & 196,304 \\
\hline 2 & 23 & 289,097 & 289,078 & 339,389 & 339,628 & 137,339 & 137,345 \\
\hline 2 & 24 & 163,137 & 163,145 & 239,825 & 239,886 & 311,389 & 311,396 \\
\hline 2 & 25 & 220,618 & 220,618 & 220,618 & 220,618 & 220,618 & 220,618 \\
\hline 2 & 26 & 311,396 & 311,389 & 239,805 & 239,901 & 163,145 & 163,137 \\
\hline 2 & 27 & 227,660 & 227,681 & 171,438 & 171,365 & 367,811 & 367,806 \\
\hline 2 & 28 & 281,740 & 281,752 & 150,769 & 150,719 & 281,752 & 281,740 \\
\hline 2 & 29 & 367,806 & 367,811 & 171,414 & 171,380 & 227,681 & 227,660 \\
\hline
\end{tabular}


Whole process consists of following steps:

- dividing the subspace enveloped by maximal workpiece and creating of auxiliary planes to facilitate the definition of individual points,

- blocking the motion of the moving platform for translation in $\mathrm{Z}$ axis,

- measuring the actual length of each arm after they reach the desired point (table 1)

\subsection{Positioning errors on arms and their influence on TCP point}

We used the module ProEngineer / Mechanism for this experiment to carry out the dynamic simulations of the mechanism motion.

Process steps (mentioned by Zabensky [7]):

- deactivation the General constrain for moving platform;

- creation of the tables with input displacements of each arm at the time. Whole process started at point 11 (figure 2). The moving platform was placed into the selected point in every sequence step. Consequently the error of $1 \mathrm{~mm}$ was applied per defined arm / arms gradually from one arm up to all of them. So at the end the error was applied to all six arms. Finally, the arms return to the home position;

- creation the tables of output values from the simulation and preparation of the output text file;

- The same procedure was applied to all other points within the workspace. The results are represented by the length deviations in $\mathrm{X}, \mathrm{Y}, \mathrm{Z}$ axis as well as the absolute space deviation of the point TCP.

\section{The measuring of the positioning errors}

The measurement is carried out for each of the 18 points separately. At the point 11 we first define a coordinate system with the same sense of marking the axes as in figure 3 . Consequently we are using the tool Measure for the measuring of the deviation between the TCP (Tool Centre Point) and defined CCS (Centre of Coordinate System) in all three axes and the overall spatial displacement.

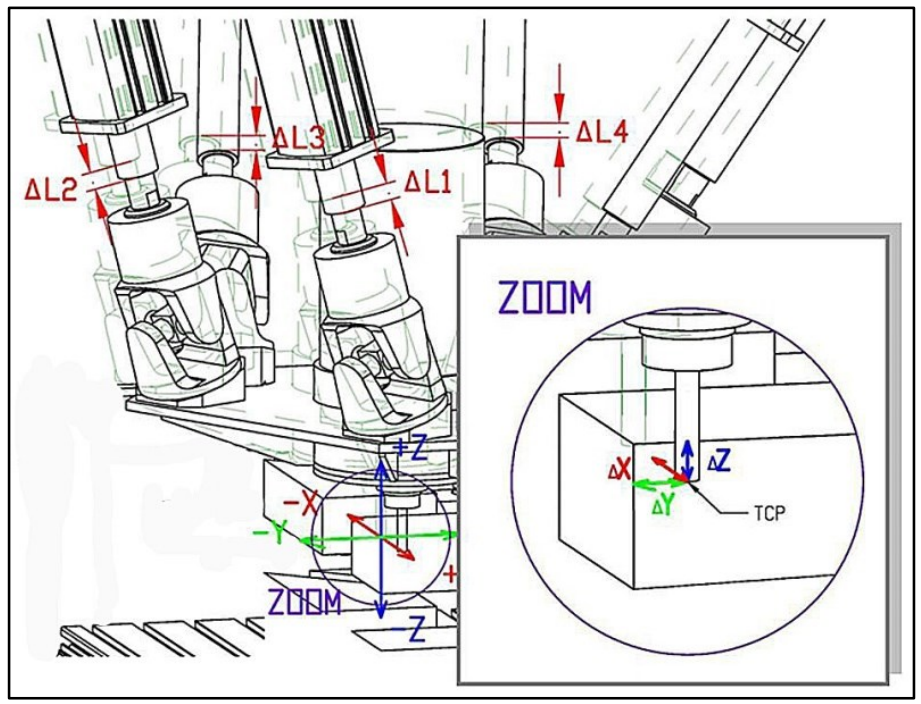

Fig. 3. Transfer of positioning errors from arms 1, 2, 3, 4 (point 11) into the position of TCP point (dashed lines shown the reference position) 


\subsection{Simulation results}

The analysis results (Table 2) can be shown in a set of graphs of output positioning errors with respect to the reference coordinate system - for each axis separately and also for total deviation. We decided for processing of obtained data in Microsoft Excel. There can be observed mainly two different influences:

- position of moving platform within the workpiece volume

- and the number of error affected arm / arms.

Table 2. Output TCP positioning errors in individual points (level 1)

\begin{tabular}{|c|c|c|c|c|c|c|c|}
\hline & \multirow{2}{*}{$\begin{array}{c}\text { Output } \\
\text { deviation } \\
(\mathrm{mm})\end{array}$} & \multicolumn{6}{|c|}{ Input positioning error $2 \mu \mathrm{m}$ applied on arm / arms number: } \\
\hline & & 1 & 1,2 & $1,2,3$ & $1,2,3,4$ & $1,2,3,4,5$ & $1,2,3,4,5,6$ \\
\hline Point: & in axis $\mathrm{X}$ & $-0,302$ & $-1,045$ & 0,232 & 2,01 & 0,672 & 0,628 \\
\hline \multirow[t]{3}{*}{11} & in axis $\mathrm{Y}$ & 0,931 & 2,066 & 1,282 & 2,082 & 0,715 & $-0,543$ \\
\hline & in axis $Z$ & $-0,615$ & $-1,51$ & $-1,082$ & $-1,589$ & $-2,454$ & $-2,15$ \\
\hline & TOTAL & 1,156 & 2,764 & 1,694 & 3,301 & 2,643 & 2,305 \\
\hline Point: & in axis $\mathrm{X}$ & 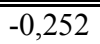 & $\begin{array}{c}-1,292 \\
\end{array}$ & 0,26 & $=1,833$ & "0,792 & 0,452 \\
\hline \multirow[t]{3}{*}{12} & in axis $Y$ & 1,076 & 2,333 & 1,522 & 2,324 & 1,071 & $-0,042$ \\
\hline & in axis $\mathrm{Z}$ & $-0,184$ & $-1,068$ & $-1,201$ & $-1,152$ & $-2,022$ & $-2,127$ \\
\hline & TOTAL & 1,120 & 2,873 & 1,956 & 3,176 & 2,421 & 2,175 \\
\hline Point: & in axis $X$ & $-0,422$ & -1,904 & "-0,112 & 101,174 & 0,458 & $-0,142$ \\
\hline \multirow[t]{3}{*}{13} & in axis $Y$ & 2,108 & 3,213 & 2,897 & 2,998 & 2,113 & 1,641 \\
\hline & in axis $Z$ & $-0,116$ & $-0,123$ & $-1,464$ & $-1,054$ & $-1,842$ & $-2,221$ \\
\hline & TOTAL & 2,153 & 3,737 & 3,248 & 3,388 & 2,840 & 2,765 \\
\hline Point: & in axis $\mathrm{X}$ & -0,58 & $\overline{-1,324}$ & $-0,176$ & 1,54 & 0,051 & $-0,01$ \\
\hline \multirow[t]{3}{*}{14} & in axis $\mathrm{Y}$ & 1,35 & 2,226 & 1,817 & 2,043 & 1,156 & $-0,413$ \\
\hline & in axis $Z$ & $-0,871$ & $-1,245$ & $-1,226$ & $-2,005$ & $-2,224$ & $-2,157$ \\
\hline & TOTAL & 1,708 & 2,874 & 2,199 & 3,250 & 2,507 & 2,196 \\
\hline Point: & in axis $X$ & $-0,132$ & $-1,175$ & 0,203 & 1,601 & 0,534 & 0,209 \\
\hline \multirow[t]{3}{*}{15} & in axis $Y$ & 1,342 & 2,312 & 1,849 & 2,354 & 1,368 & $-0,082$ \\
\hline & in axis $\mathrm{Z}$ & $-0,411$ & $-0,822$ & $-1,17$ & $-1,567$ & $-1,927$ & $-2,325$ \\
\hline & TOTAL & 1,410 & 2,721 & 2,197 & 3,250 & 2,423 & 2,336 \\
\hline Point: & in axis $\mathrm{X}$ & \begin{tabular}{c|}
$-0,054$ \\
\end{tabular} & $-1,512$ & 0,173 & 101,322 & 0,575 & \begin{tabular}{c|}
0,018 \\
\end{tabular} \\
\hline \multirow[t]{3}{*}{16} & in axis $Y$ & 1,538 & 2,232 & 2,125 & 2,461 & 1,725 & 0,462 \\
\hline & in axis $Z$ & 0,115 & $-0,201$ & $-0,967$ & $-0,944$ & $-1,337$ & $-2,201$ \\
\hline & TOTAL & 1,543 & 2,703 & 2,341 & 2,949 & 2,257 & 2,249 \\
\hline Point: & in axis $\mathrm{X}$ & $-0,622$ & $-1,428$ & $\begin{array}{c}-0,381 \\
\end{array}$ & 101,154 & $\begin{array}{c}-0,362 \\
\end{array}$ & $-0,425$ \\
\hline \multirow[t]{3}{*}{17} & in axis $\mathrm{Y}$ & 1,617 & 2,245 & 2,134 & 2,352 & 1,601 & $-0,353$ \\
\hline & in axis $Z$ & $-1,009$ & $-0,944$ & $-1,275$ & $-2,334$ & $-2,101$ & $-2,159$ \\
\hline & TOTAL & 2,005 & 2,823 & 2,515 & 3,509 & 2,666 & 2,229 \\
\hline Point: & in axis $\mathrm{X}$ & $-0,348$ & $-1,49$ & $-0,219$ & 1,066 & $-0,076$ & $-0,431$ \\
\hline \multirow[t]{3}{*}{18} & in axis $\mathrm{Y}$ & 1,818 & 2,589 & 2,466 & 2,553 & 1,821 & 0,005 \\
\hline & in axis $\mathrm{Z}$ & $-0,548$ & $-0,442$ & $-1,107$ & $-1,671$ & $-1,545$ & $-2,222$ \\
\hline & TOTAL & 1,930 & 3,020 & 2,712 & 3,232 & 2,389 & 2,263 \\
\hline Point: & in axis $\mathrm{X}$ & - -0,145 & \begin{tabular}{c|}
$-1,708$ \\
\end{tabular} & $-0,144$ & 0,908 & 0,125 & $-0,471$ \\
\hline \multirow[t]{3}{*}{19} & in axis $\mathrm{Y}$ & 2,007 & 2,792 & 2,699 & 2,845 & 2,111 & 0,398 \\
\hline & in axis $Z$ & $-0,024$ & 0,249 & $-0,803$ & $-1,102$ & $-1,087$ & $-2,245$ \\
\hline & TOTAL & 2,012 & 3,282 & 2,820 & 3,183 & 2,378 & 2,328 \\
\hline
\end{tabular}


All positioning errors in each point of level 1 were compared with the positioning errors in reference point 15 (middle of the worskspace). On this basis were created the graphs (figure 4, 5, 6 and 7) represented the relation between the point TCP from the vertical axis of the machine tool (workspace). The positioning errors are divided according to the axes $\mathrm{X}, \mathrm{Y}, \mathrm{Z}$ and total space distance.

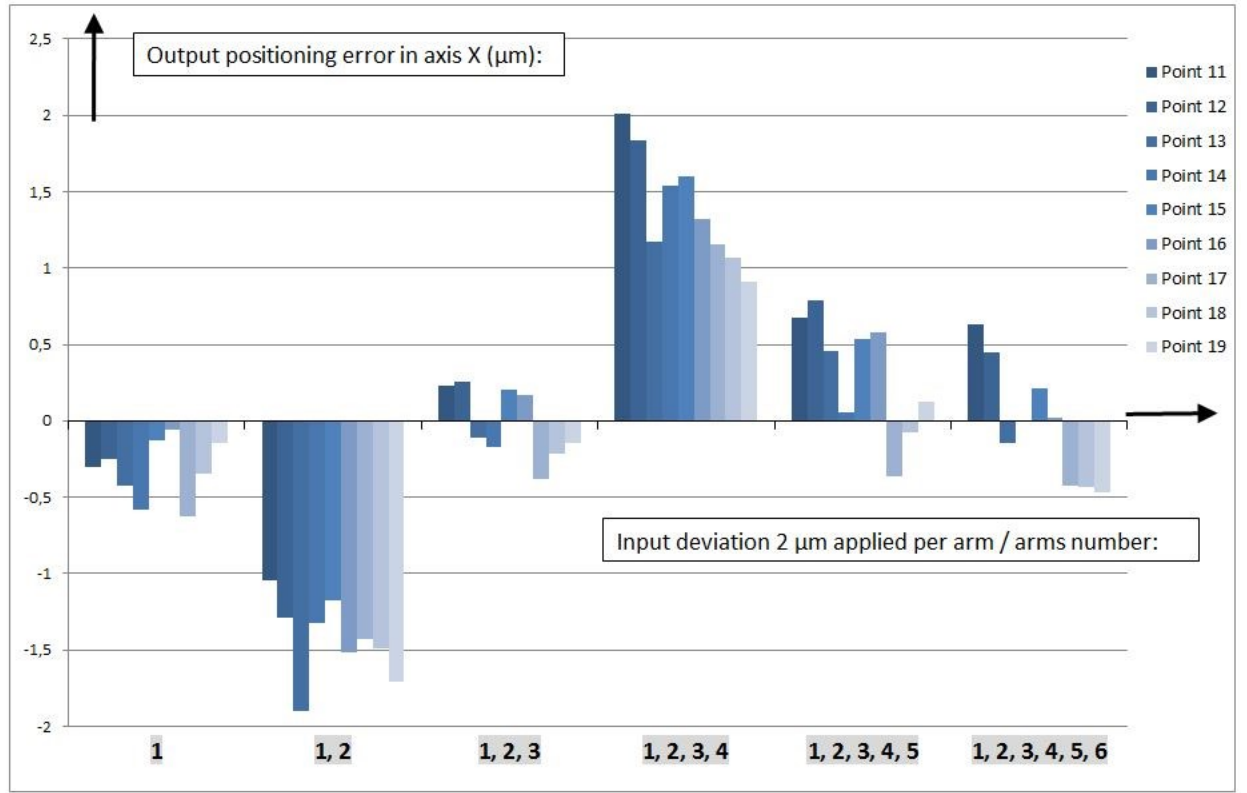

Fig. 4. Graph of output positioning error of point TCP in axis X (level 1)

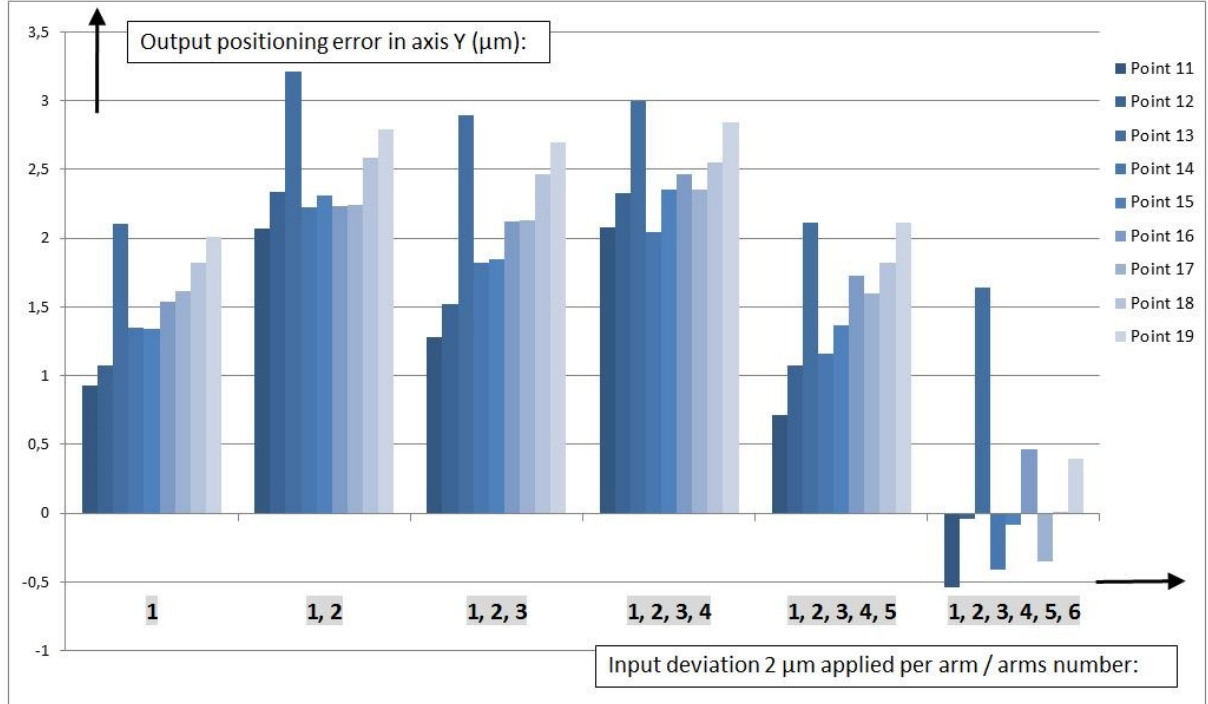

Fig. 5. Graph of output positioning error of point TCP in axis Y (level 1) 


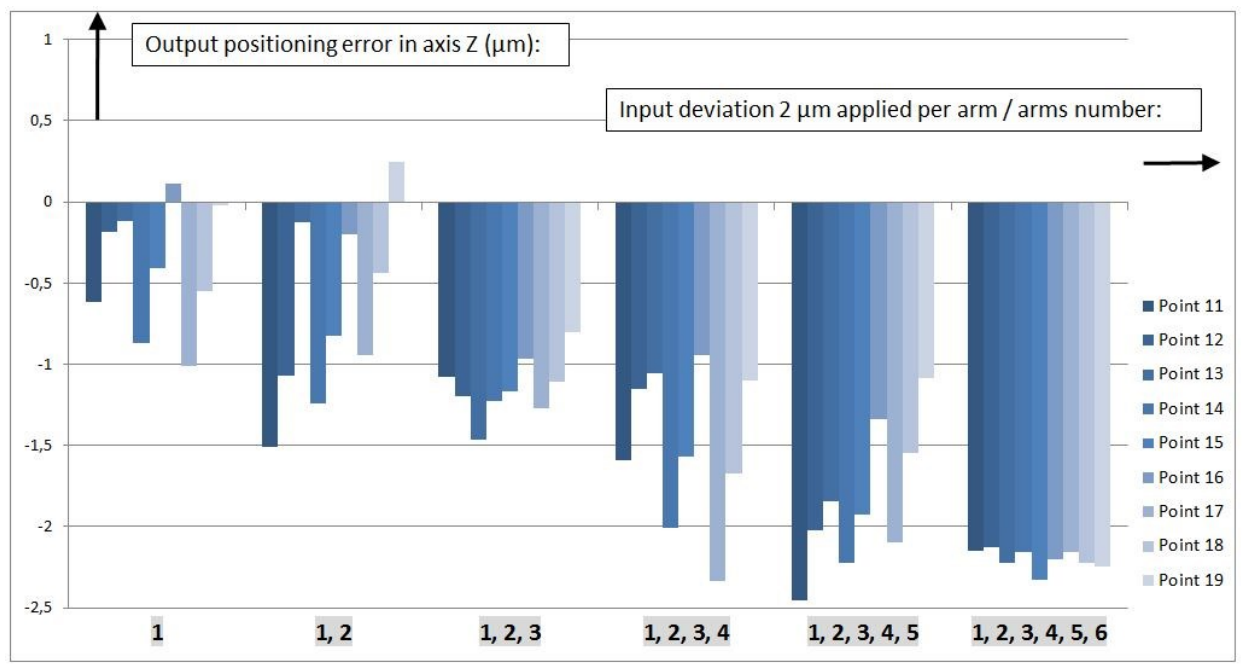

Fig. 6. Graph of output positioning error of point TCP in axis Z (level 1)

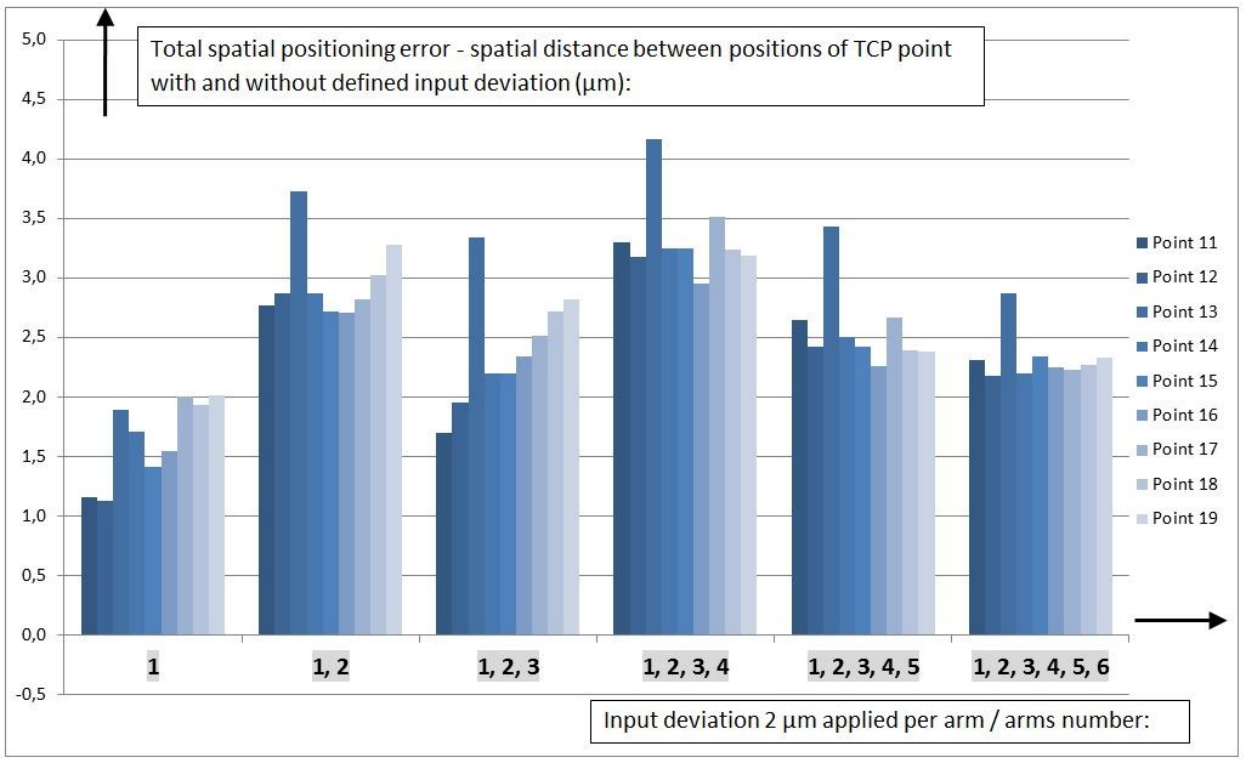

Fig. 7. Graph of total spatial output positioning error of point TCP (level 1)

\section{Conclusions}

In recent years, due to fewer driving elements, lower cost, and more compact structure, lower-mobility parallel mechanisms have shown great potential for application, and have has gradually attracted the attention of scholars both at home and abroad [8].

The main aim of this article was to demonstrate the uncertainties influence of telescopic arm control in case of mechanism with parallel kinematic structure called Hexapod ZU. There can be visible also the difference between measurement of accuracy on conventional machines and machines with parallel kinematics. The accuracy in case of parallel machines 
is much harder to achieve and to define. This was also the main reason why we decided to analyse the relationship between the accuracy in actuators and in moving platform.

On this analysis we can conclude that:

- the maximum deviation in $\mathrm{X}$ and $\mathrm{Y}$ axis as well as the final deviation in both levels happened by errors applied on arms 1, 2 or 1, 2, 3, 4 (together);

- the deviation in $\mathrm{X}$ and $\mathrm{Y}$ axis is close to zero in both levels when the errors is applied on all arms;

- the value of deviation in the Z-axis in both levels is directly proportional to the number of "non-precision" controlled arms;

- the impact of the distance from the vertical axis of the machine is in level 1 greater than in level 2;

- it's important to know the deviation in the Z-axis when we measure the deviation of circular interpolation in the XY plane for proper assessment of the results.

Therefore we approve that the size of the final TCP point deviation is not affected only by the error on each arm but by the current position of the platform as well. In level 1 were observed in all axes higher deviations from the reference point. From this we can conclude, that the size of deviations is changing with respect to the position of moving platform within the workspace.

The authors disclosed receipt of the following financial support for the publication of this article: This work is partly supported by the project VEGA 1/0504/17: Research and development of methods for multi-criteria diagnosis of $\mathrm{CNC}$ machine tools' accuracy.

\section{References}

1. V. Bulej, Mechanization and automation equipment for processing: Innovative and non-standard applications of mechanisms with parallel kinematic structure. (Publ. House Alma Mater, Cluj-Napoca, 2015)

2. J. Uricek, et al., Design of Machine Tool with Parallel Kinematic Structure. Sci. Reports - J. of Univ. of Appl. Sci. Mittweida 21, 36-39 (2011)

3. V. Bulej et al., The modelling of mechanism with parallel kinematic structure in software Matlab/Simulink. Transactions of the VSB 62, 1-8 (2016)

4. B. Leitner, M. Vaško, Design and Modelling of Tank Wagon Assembly Operations in CAM Environment. Transport Means 2015 I \& II, $87-90$ (2015)

5. M. Krajcovic, et al., Intelligent manufacturing systems in concept of digital factory. Communications : Sci. Letters of the Uni. of Zilina, 15, 77-87 (2013)

6. G. Palmieri, On the positioning error of a 2-DOF spherical parallel wrist with flexible links and joints - an FEM approach. Mechanical Sci. 6, 9-14 (2015)

7. M. Zabensky, Evaluation of selected operational properties of machine tool prototype with parallel kinematic structure. Diploma thesis, Zilina (2013)

8. X. Chen et al., Rigid Dynamic Model and Analysis of 5-DOF Parallel Mechanism. Int J Adv Robot Syst, 1-9 (2015) 